\title{
Continuous Inversion Formulas for Multi-Dimensional Stockwell Transforms
}

\author{
L. Riba ${ }^{1}, \mathrm{M}$ W. Wong ${ }^{2}$. \\ ${ }^{1}$ Dipartimento di Matematica, Università di Torino, via Carlo Alberto 10, \\ 10123 Torino, Italy \\ ${ }^{2}$ Department of Mathematics and Statistics, York University, 4700 Keele Street, \\ Toronto, Ontario M3J 1P3, Canada
}

\begin{abstract}
Stockwell transforms as hybrids of Gabor transforms and wavelet transforms have been studied extensively. We introduce in this paper multi-dimensional Stockwell transforms that include multi-dimensional Gabor transforms as special cases. Continuous inversion formulas for multi-dimensional Stockwell transforms are proved.
\end{abstract}

Keywords and phrases: Gabor transforms, wavelet transforms, Stockwell transforms, continuous inversion formulas

Mathematics Subject Classification: 42C40, 47G10

\section{Introduction}

Let us recall that for a signal $f$ in $L^{2}(\mathbb{R})$, the Gabor transform or the short-time Fourier transform $G_{\varphi} f$ of $f$ with respect to a window $\varphi$ in $L^{1}(\mathbb{R}) \cap L^{2}(\mathbb{R})$ is defined by

$$
\left(G_{\varphi} f\right)(b, \xi)=(2 \pi)^{-1 / 2} \int_{-\infty}^{\infty} e^{-i x \xi} f(x) \overline{\varphi(x-b)} d x
$$

for all $b, \xi \in \mathbb{R}$.

Let us note that

$$
\left(G_{\varphi} f\right)(b, \xi)=(2 \pi)^{-1 / 2}\left(f, M_{\xi} T_{-b} \varphi\right)_{L^{2}(\mathbb{R})}
$$

for all $b, \xi \in \mathbb{R}^{n}$, where $(,)_{L^{2}(\mathbb{R})}$ is the inner product in $L^{2}(\mathbb{R}), M_{\xi}$ and $T_{-b}$ are the modulation operator and the translation operator given by

$$
\left(M_{\xi} h\right)(x)=e^{i x \xi} h(x),
$$

and

$$
\left(T_{-b} h\right)(x)=h(x-b)
$$

${ }^{*}$ Corresponding author. E-mail: mwwong@mathstat.yorku.ca, This research has been supported by a discovery grant from the Natural Sciences and Engineering Research Council of Canada. 
for all measurable functions $h$ on $\mathbb{R}$ and all $x$ in $\mathbb{R}$. We call the function $M_{\xi} T_{-b} \varphi$ the Gabor wavelet generated from $\varphi$ by translation $T_{-b}$ and modulation $M_{\xi}$.

The usefulness of the Gabor wavelets in signal analysis is enhanced by the following resolution of the identity formula, which allows the reconstruction of a signal from its Gabor transform.

Theorem 1.1. Suppose that $\|\varphi\|_{2}=1$, where \|\|$_{2}$ is the norm in $L^{2}(\mathbb{R})$. Then for all $f$ and $g$ in $L^{2}(\mathbb{R})$,

$$
(f, g)_{L^{2}(\mathbb{R})}=\int_{-\infty}^{\infty} \int_{-\infty}^{\infty}\left(G_{\varphi} f\right)(b, \xi) \overline{\left(G_{\varphi} g\right)(b, \xi)} d b d \xi
$$

Another way of looking at Theorem 1.1 is that for all $f$ in $L^{2}(\mathbb{R})$,

$$
f=(2 \pi)^{-1} \int_{-\infty}^{\infty} \int_{-\infty}^{\infty}\left(f, M_{\xi} T_{-b} \varphi\right)_{L^{2}(\mathbb{R})} M_{\xi} T_{-b} \varphi d b d \xi
$$

The formula in Theorem 1.1 is also known as a continuous inversion formula for the Gabor transform. In signal analysis, the term $\left(G_{\varphi} f\right)(b, \xi)$ gives the time-frequency content of a signal $f$ at time $b$ and frequency $\xi$ by placing the window $\varphi$ at time $b$. The drawback here is that a window of fixed width is used for all time $b$. It is more accurate and desirable if we can have an adaptive window that gives a wide window for low frequency and a narrow window for high frequency. That this can be done comes from familiarity with the wavelet transform that we now recall.

Let $\varphi \in L^{2}(\mathbb{R})$ be such that

$$
\int_{-\infty}^{\infty} \frac{|\hat{\varphi}(\xi)|^{2}}{|\xi|} d \xi<\infty
$$

where $\hat{\varphi}$ is the Fourier transform of $\varphi$ and is sometimes denoted by $\mathcal{F} \varphi$. The convention that we use in this paper is that the Fourier transform $\hat{f}$ of a function $f$ in $L^{1}\left(\mathbb{R}^{n}\right)$ is defined by

$$
\hat{f}(\xi)=(2 \pi)^{-n / 2} \int_{\mathbb{R}^{n}} e^{-i x \cdot \xi} f(x) d x, \quad \xi \in \mathbb{R}^{n} .
$$

Such a function $\varphi$ is said to satisfy the admissibility condition and is sometimes called the mother affine wavelet. The adjective affine comes from the connection with the affine group that is the underpinning of the wavelet transforms. See Chapter 18 of [16] in this connection.

Let $\varphi \in L^{2}(\mathbb{R})$ be a mother affine wavelet. Then for all $b$ in $\mathbb{R}$ and $a$ in $\mathbb{R} \backslash\{0\}$, we define the affine wavelet $\varphi_{b, a}$ by

$$
\varphi_{b, a}(x)=\frac{1}{\sqrt{|a|}} \varphi\left(\frac{x-b}{a}\right)
$$

for all $x$ in $\mathbb{R}$. We note that $\varphi_{b, a}$ is generated from the function $\varphi$ by translation and dilation. To put the formula in perspective, let $b \in \mathbb{R}$ and let $a \in \mathbb{R} \backslash\{0\}$. Then we see that the wavelet $\varphi_{b, a}$ can be expressed as

$$
\varphi_{b, a}=T_{-b} D_{1 / a} \varphi
$$

where $D_{1 / a}$ is the dilation operator defined by

$$
\left(D_{1 / a} h\right)(x)=\frac{1}{\sqrt{|a|}} h\left(\frac{x}{a}\right)
$$

for all measurable functions $h$ on $\mathbb{R}$ and and all $x$ in $\mathbb{R}$.

Let $\varphi$ be a mother affine wavelet. Then the wavelet transform $\Omega_{\varphi} f$ of a function $f$ in $L^{2}(\mathbb{R})$ is defined to be the function on $\mathbb{R} \times \mathbb{R} \backslash\{0\}$ by

$$
\left(\Omega_{\varphi} f\right)(b, a)=\left(f, \varphi_{b, a}\right)_{L^{2}(\mathbb{R})}
$$

for all $b$ in $\mathbb{R}$ and $a$ in $\mathbb{R} \backslash\{0\}$. The nucleus of the analysis of the wavelet transform is the following resolution of the identity formula, which is a continuous inversion formula. 
Theorem 1.2. Let $\varphi$ be a mother affine wavelet. Then for all functions $f$ and $g$ in $L^{2}(\mathbb{R})$,

$$
(f, g)_{L^{2}(\mathbb{R})}=\frac{1}{c_{\varphi}} \int_{-\infty}^{\infty}\left(f, \varphi_{b, a}\right)_{L^{2}(\mathbb{R})}\left(\varphi_{b, a}, g\right)_{L^{2}(\mathbb{R})} \frac{d b d a}{a^{2}},
$$

where

$$
c_{\varphi}=2 \pi \int_{-\infty}^{\infty} \frac{|\hat{\varphi}(\xi)|^{2}}{|\xi|} d \xi
$$

Remark 1.3. It can also be proved that a necessary condition for the continuous inversion formula to hold is that $\varphi$ has to be a mother affine wavelet. Indeed, suppose that

$$
\int_{-\infty}^{\infty} \int_{-\infty}^{\infty}\left(f, \varphi_{b, a}\right)_{L^{2}(\mathbb{R})}\left(\varphi_{b, a}, g\right)_{L^{2}(\mathbb{R})} \frac{d b d a}{a^{2}}
$$

exists for all $f$ and $g$ in $L^{2}(\mathbb{R})$. Then, letting $f=g=\varphi$, we get

$$
\int_{-\infty}^{\infty}\left|\left(\varphi, \varphi_{b, a}\right)_{L^{2}(\mathbb{R})}\right|^{2} \frac{d b d a}{a^{2}}<\infty
$$

which can be shown to be the same as

$$
\int_{-\infty}^{\infty} \frac{|\hat{\varphi}(\xi)|^{2}}{|\xi|} d \xi<\infty
$$

Details can be found in [5] and [16].

The resolution of the identity formula leads to the reconstruction formula, which says that

$$
f=\frac{1}{c_{\varphi}} \int_{-\infty}^{\infty}\left(f, \varphi_{b, a}\right)_{L^{2}(\mathbb{R})} \varphi_{b, a} \frac{d b d a}{a^{2}}
$$

for all $f$ in $L^{2}(\mathbb{R})$. In other words, we have a continuous inversion formula for the signal $f$ from a knowledge of its time-scale spectrum.

As in the case of the Gabor transform, there is a window $\varphi_{b, a}$ in the wavelet transform. Unlike the case of the Gabor transform, the window $\varphi_{b, a}$ is adjustable in the sense that it is narrow if the scale $a$ is small and wide if the scale $a$ is big.

Now, let $\varphi \in L^{1}(\mathbb{R}) \cap L^{2}(\mathbb{R})$. Then, combining the merits of the Gabor transform and the wavelet transform, the Stockwell transform $S_{\varphi} f$ with window $\varphi$ of a signal $f$ in $L^{2}(\mathbb{R})$ is defined by

$$
\left(S_{\varphi} f\right)(b, \xi)=(2 \pi)^{-1 / 2}|\xi| \int_{-\infty}^{\infty} e^{-i x \xi} f(x) \overline{\varphi(\xi(x-b))} d x
$$

for all $b \in \mathbb{R}$ and $\xi \in \mathbb{R} \backslash\{0\}$. We note that for all $f$ in $L^{2}(\mathbb{R})$, all $b$ in $\mathbb{R}$ and all $\xi$ in $\mathbb{R} \backslash\{0\}$,

$$
\left(S_{\varphi} f\right)(b, \xi)=\left(f, \varphi^{b, \xi}\right)_{L^{2}(\mathbb{R})},
$$

where

$$
\varphi^{b, \xi}=(2 \pi)^{-1 / 2} M_{\xi} T_{-b} \tilde{D}_{\xi} \varphi
$$

Here, the dilation operator $\tilde{D}_{\xi}$ is defined by

$$
\left(\tilde{D}_{\xi} h\right)(x)=|\xi| h(\xi x)
$$

for all $x$ in $\mathbb{R}$ and all measurable functions $h$ on $\mathbb{R}$. Besides the modulation with respect to frequency $\xi$, a notable feature in the Stockwell transform is the normalizing factor in the dilation operator, which is $|\cdot|$ in lieu of $|\cdot|^{1 / 2}$ as in the case of the wavelet transform, and is the mathematical underpinning of the absolutely referenced phase information in [13] formulated in Theorem 1.5. These features distinguish the Stockwell transform from the wavelet transform. Notwithstanding these differences, we have the following formula in [13] relating the Stockwell transform to the Morlet wavelet transform $\Omega_{\psi}$. 
Theorem 1.4. For all $f$ in $L^{2}(\mathbb{R})$,

$$
\left(S_{\varphi} f\right)(b, \xi)=(2 \pi)^{-1 / 2} e^{-i b \xi} \sqrt{|\xi|}\left(\Omega_{\psi} f\right)(b, 1 / \xi)
$$

for all $b \in \mathbb{R}$ and $\xi \in \mathbb{R} \backslash\{0\}$, where

$$
\psi(x)=e^{i x} \varphi(x)
$$

for all $x$ in $\mathbb{R}$.

The Stockwell transform is closely related to the wave packet transform of Cordoba and Fefferman [4], which also involves a combination of translations, modulations and dilations. It should also be mentioned that transforms closely related to the wavelet transforms and the metaplectic representation abound and can be found in the monographs $[1,7]$, and the works $[3,14]$.

Of particular importance in the Stockwell transform is the phase correction in the preceding formula given by $e^{-i b \xi}$, which is caused by the phase function $e^{-i x \xi}$ inside the integral defining the Stockwell transform. It is crucial to note that this function picks out the frequency to be localized, but is not translated with respect to time $b$ as is always done for the Morlet wavelet transform [9]. To see the full significance of this, we note that in real-life applications, signals $f$ and windows $\varphi$ are real-valued functions. Therefore information about the phase $\arg \left(S_{\varphi} f\right)(b, \xi)$ of the Stockwell transform $\left(S_{\varphi} f\right)(b, \xi)$ at time $b$ and frequency $\xi$ comes from the term $e^{-i x \xi}$ at time $b=0$. But in the case of the Morlet wavelet transform, the phase information is obtained by referencing the windowed signal $f$ with respect to $e^{-i(x-b) \xi}$. This is precisely the absolutely referenced phase information in [13] and is responsible for the continuous inversion formula in Theorem 1.5 given later. Another point is that the Stockwell transform is reminiscent of the Morlet wavelet transform, but the applicability of the computational techniques available for the Morlet wavelet transforms is undermined by the inversion $a=1 / \xi$.

The Stockwell transform has recently been used in geophysics $[6,13]$ and in medical imaging $[8,18]$. More recent applications in imaging are in $[10,11]$. In view of its versatility, an attempt in understanding the mathematical underpinnings of the Stockwell transform is worthwhile. The following continuous inversion formula for the Stockwell transform can be found in [13] and [17] for the case when

$$
\varphi(x)=e^{-\pi x^{2}}
$$

for all $x$ in $\mathbb{R}$.

Theorem 1.5. Let $\varphi \in L^{1}(\mathbb{R}) \cap L^{2}(\mathbb{R})$ be such that

$$
\int_{-\infty}^{\infty} \varphi(x) d x=1
$$

Then for all $f$ in $L^{2}(\mathbb{R})$,

$$
f=\mathcal{F}^{-1} A S_{\varphi} f
$$

where $\mathcal{F}^{-1}$ is the inverse Fourier transform and $A$ is the time average operator given by

$$
(A F)(\xi)=\int_{-\infty}^{\infty} F(b, \xi) d b
$$

for all $\xi$ in $\mathbb{R}$ and all measurable functions $F$ on $\mathbb{R} \times \mathbb{R}$, provided that the integral exists.

Another continuous inversion formula for the Stockwell transform akin to the continuous inversion formulas for the Gabor transform and the wavelet transform is given by the following theorem.

Theorem 1.6. Let $\varphi \in L^{2}(\mathbb{R})$ be such that

$$
\int_{-\infty}^{\infty} \frac{|\hat{\varphi}(\xi-1)|^{2}}{|\xi|} d \xi<\infty
$$


Then for all $f$ and $g$ in $L^{2}(\mathbb{R})$,

$$
(f, g)_{L^{2}(\mathbb{R})}=\frac{1}{c_{\varphi}} \int_{-\infty}^{\infty} \int_{-\infty}^{\infty}\left(f, \varphi^{b, \xi}\right)_{L^{2}(\mathbb{R})}\left(\varphi^{b, \xi}, g\right)_{L^{2}(\mathbb{R})} \frac{d b d \xi}{|\xi|}
$$

where

$$
c_{\varphi}=\int_{-\infty}^{\infty} \frac{|\hat{\varphi}(\xi-1)|^{2}}{|\xi|} d \xi .
$$

Remark 1.7. Theorem 1.6 tells us that every signal $f$ can be resconstructed from its Stockwell spectrum by means of the formula

$$
f=\frac{1}{c_{\varphi}} \int_{-\infty}^{\infty} \int_{-\infty}^{\infty}\left(f, \varphi^{b, \xi}\right)_{L^{2}(\mathbb{R})} \varphi^{b, \xi} \frac{d b d \xi}{|\xi|} .
$$

Using exactly the same argument given in Remark 1.3 for the wavelet transform, we see that a necessary condition for the continuous inversion formula to hold for the Stockwell transform is that

$$
\int_{-\infty}^{\infty} \frac{|\hat{\varphi}(\xi-1)|^{2}}{|\xi|} d \xi<\infty
$$

This means that $\hat{\varphi}(-1)=0$ whenever $\hat{\varphi}$ is continuous at -1 . So, it is important to observe that the Gaussian window used exclusively for the Stockwell transform in the literature is not admissible.

We introduce in this paper $n$-dimensional Stockwell transforms, $n>1$, and give the corresponding continuous inversion formulas analogous to Theorems 1.5 and 1.6 for the one-dimensional Stockwell transform. In view of the needs of analyzing data that depend on many parameters and of modern developments of physics in which dimensions more than four are envisaged, it is reasonable to expect that an $n$-dimensional multi-scale integral transform may be useful. The $n$-dimensional Stockwell transforms are also the basic ingredients of filters in the analysis of signals and images, also known as localization operators in [16] of which the mapping properties are studied in detail.

In Section 2 we define $n$-dimensional Stockwell transforms and we prove a resolution of the identity formula under some assumptions. In Section 3 we introduce the most simplistic $n$-dimensional Stockwell transforms, which are in fact $n$-dimensional Gabor transforms. We give for them the continuous inversion formula that is the analog of the one in Theorem 1.1 for one-dimensional Gabor transforms. In Section 4 we introduce the non-isotropic $n$-dimensional Stockwell transforms and give for them the continuous inversion formula. In a nutshell, we have modified through appropriate normalizations in the $L^{2}$ norm the Stockwell transform so that it becomes the Gabor transform in the preceding section. The results and examples in this section are known as special cases of reproducing formulas obtained from the metaplectic representation in Chapter 4 of [7] and in [3]. In Section 5 we give some examples of matrices that guarantee the resolution of the identity formula for the associated Stockwell transform. Some concluding comments are given in Section 6.

The results in this paper extend those for the two-dimensional Stockwell transforms in [12].

\section{Multi-Dimensional Stockwell Transforms}

For all $\xi \in \mathbb{R}^{n}$, let $A_{\xi}$ be an invertible $n \times n$ matrix, i.e.,

$$
A_{\xi}=\left(a_{i j}(\xi)\right)_{1 \leq i, j \leq n} .
$$

Let $\varphi \in L^{1}\left(\mathbb{R}^{n}\right) \cap L^{2}\left(\mathbb{R}^{n}\right)$ be such that

$$
\int_{\mathbb{R}^{n}} \varphi(x) d x=1 .
$$


Then the $n$-dimensional Stockwell transform $S_{\varphi} f$ of a signal $f$ in $L^{2}\left(\mathbb{R}^{n}\right)$ with respect to the window $\varphi$ is given by

$$
\left(S_{\varphi} f\right)(b, \xi)=(2 \pi)^{-n / 2}\left|\operatorname{det} A_{\xi}\right| \int_{\mathbb{R}^{n}} e^{-i x \cdot \xi} f(x) \overline{\varphi\left(A_{\xi}(x-b)\right)} d x
$$

for all $b$ in $\mathbb{R}^{n}$ and all $\xi$ in $\mathbb{R}^{n}$. We first note that the inversion formula in Theorem 1.5 for the multidimensional Stockwell transform $S_{\varphi}$ holds. Indeed, for all $\xi \in \mathbb{R}^{n}$,

$$
\begin{aligned}
\int_{\mathbb{R}^{n}}\left(S_{\varphi} f\right)(b, \xi) d b & =\frac{\left|\operatorname{det} A_{\xi}\right|}{(2 \pi)^{n / 2}} \int_{\mathbb{R}^{n}} \int_{\mathbb{R}^{n}} e^{-i x \cdot \xi} f(x) \overline{\varphi\left(A_{\xi}(x-b)\right)} d x d b \\
& =\frac{\left|\operatorname{det} A_{\xi}\right|}{(2 \pi)^{n / 2}} \int_{\mathbb{R}^{n}} e^{-i x \cdot \xi} f(x)\left[\int_{\mathbb{R}^{n}} \overline{\varphi\left(A_{\xi}(x-b)\right)} d b\right] d x \\
& =(2 \pi)^{-n / 2} \int_{\mathbb{R}^{n}} e^{-i x \cdot \xi} f(x)\left[\int_{\mathbb{R}^{n}} \varphi(y) d y\right] d x \\
& =(2 \pi)^{-n / 2} \int_{\mathbb{R}^{n}} e^{-i x \cdot \xi} f(x) d x \\
& =\hat{f}(\xi) .
\end{aligned}
$$

Thus,

$$
f=\mathcal{F}^{-1} A S_{\varphi} f
$$

where $\mathcal{F}^{-1}$ is the inverse Fourier transform and $A$ is the operator given by

$$
(A F)(\xi)=\int_{\mathbb{R}^{n}} F(b, \xi) d b
$$

for all $\xi$ in $\mathbb{R}^{n}$ and all measurable functions $F$ on $\mathbb{R}^{n} \times \mathbb{R}^{n}$, provided that the integral exists.

We note also that the $n$-dimensional Stockwell transform $S_{\varphi}$ can be written as

$$
\left(S_{\varphi} f\right)(b, \xi)=\left(f, \varphi^{b, \xi, A_{\xi}}\right)_{L^{2}\left(\mathbb{R}^{n}\right)}
$$

for all $b \in \mathbb{R}^{n}$ and $\xi \in \mathbb{R}^{n}$, where

$$
\varphi^{b, \xi, A_{\xi}}=(2 \pi)^{-n / 2} M_{\xi} T_{-b} D_{A_{\xi}} \varphi
$$

and $M_{\xi}, T_{-b}$ and $D_{A_{\xi}}$ are the modulation operator, the translation operator and the dilation operator given, respectively, by

$$
\begin{gathered}
\left(M_{\xi} \varphi\right)(x)=e^{i x \cdot \xi} \varphi(x), \\
\left(T_{-b} \varphi\right)(x)=\varphi(x-b)
\end{gathered}
$$

and

$$
\left(D_{A_{\xi}} \varphi\right)(x)=\left|\operatorname{det} A_{\xi}\right| \varphi\left(A_{\xi} x\right)
$$

for all $x$ in $\mathbb{R}^{n}$. It is easy to see that for all $\eta$ in $\mathbb{R}^{n}$,

$$
\left(M_{\xi} \varphi\right)^{\wedge}(\eta)=\left(T_{-\xi} \hat{\varphi}\right)(\eta)
$$

and

$$
\left(T_{-b} \varphi\right)^{\wedge}(\eta)=\left(M_{-b} \hat{\varphi}\right)(\eta)
$$

The Fourier transform of $D_{A_{\xi}} \varphi$ is given in the following proposition.

Proposition 2.1. For all $\eta$ in $\mathbb{R}^{n}$,

$$
\left(D_{A_{\xi}} \varphi\right)^{\wedge}(\eta)=\left|\operatorname{det} A_{\xi}\right|\left(D_{\left(A_{\xi}^{-1}\right)^{t}} \hat{\varphi}\right)(\eta)
$$

for all $\xi$ in $\mathbb{R}^{n} \backslash\{0\}$ and all $\eta$ in $\mathbb{R}^{n}$, where $\left(A_{\xi}^{-1}\right)^{t}$ is the transpose of the inverse of the matrix $A_{\xi}$. 
Proof. Let $\eta \in \mathbb{R}^{n}$. Then

$$
\begin{aligned}
\left(D_{A_{\xi}} \varphi\right)^{\wedge}(\eta) & =(2 \pi)^{-n / 2} \int_{\mathbb{R}^{n}} e^{-i x \cdot \eta}\left(D_{A_{\xi}} \varphi\right)(x) d x \\
& =(2 \pi)^{-n / 2} \int_{\mathbb{R}^{n}} e^{-i x \cdot \eta}\left|\operatorname{det} A_{\xi}\right| \varphi\left(A_{\xi} x\right) d x \\
& =(2 \pi)^{-n / 2} \int_{\mathbb{R}^{n}} e^{-i\left(A_{\xi}^{-1} x\right) \cdot \eta} \varphi(x) d x \\
& =(2 \pi)^{-n / 2} \int_{\mathbb{R}^{n}} e^{-i x \cdot\left(\left(A_{\xi}^{-1}\right)^{t} \eta\right)} \varphi(x) d x \\
& =\hat{\varphi}\left(\left(A_{\xi}^{-1}\right)^{t} \eta\right) \\
& \left.=\left|\operatorname{det}\left(A_{\xi}^{-1}\right)^{t}\right|^{-1} D_{\left(A_{\xi}^{-1}\right)^{t}} \hat{\varphi}\right)(\eta) \\
& =\left|\operatorname{det} A_{\xi}\right|\left(D_{\left(A_{\xi}^{-1}\right)^{t}} \hat{\varphi}\right)(\eta) .
\end{aligned}
$$

Proposition 2.2. For all $f$ in $L^{2}\left(\mathbb{R}^{n}\right)$,

$$
\left(S_{\varphi} f\right)(b, \xi)=e^{-i b \cdot \xi}\left(\mathcal{F}^{-1} f_{\xi, A_{\xi}}\right)(b)
$$

for all $b \in \mathbb{R}^{n}$ and $\xi \in \mathbb{R}^{n} \backslash\{0\}$, where

$$
f_{\xi, A_{\xi}}(\eta)=\hat{f}(\eta) \overline{\hat{\varphi}\left(\left(A_{\xi}^{-1}\right)^{t}(\eta-\xi)\right)}
$$

for all $\eta \in \mathbb{R}^{n}$.

Proof. For all $\xi$ in $\mathbb{R}^{n} \backslash\{0\}$ and $\eta$ in $\mathbb{R}^{n}$, we get

$$
\begin{aligned}
& \left(\varphi^{b, \xi, A_{\xi}}\right)^{\wedge}(\eta) \\
= & (2 \pi)^{-n / 2}\left(M_{\xi} T_{-b} D_{A_{\xi}} \varphi\right)^{\wedge}(\eta) \\
= & (2 \pi)^{-n / 2} T_{-\xi}\left(T_{-b} D_{A_{\xi}} \varphi\right)^{\wedge}(\eta) \\
= & (2 \pi)^{-n / 2} T_{-\xi} M_{-b}\left(D_{A_{\xi}} \varphi\right)^{\wedge}(\eta) \\
= & (2 \pi)^{-n / 2} T_{-\xi} M_{-b}\left|\operatorname{det} A_{\xi}\right|\left(D_{\left(A_{\xi}^{-1}\right)^{t}} \hat{\varphi}\right)(\eta) \\
= & (2 \pi)^{-n / 2} e^{-i b \cdot(\eta-\xi)} \hat{\varphi}\left(\left(A_{\xi}^{-1}\right)^{t}(\eta-\xi)\right) .
\end{aligned}
$$

Thus, by Plancherel's formula, we obtain for all $b$ in $\mathbb{R}^{n}$ and $\xi$ in $\mathbb{R}^{n} \backslash\{0\}$,

$$
\begin{aligned}
\left(S_{\varphi} f\right)(b, \xi) & =\left(f, \varphi^{b, \xi, A_{\xi}}\right)_{L^{2}\left(\mathbb{R}^{n}\right)} \\
& =\int_{\mathbb{R}^{n}} f(x) \overline{\varphi^{b, \xi, A_{\xi}(x)}} d x \\
& =\int_{\mathbb{R}^{n}} \hat{f}(\eta) \overline{\left(\varphi^{\left.b, \xi, A_{\xi}\right)^{\wedge}(\eta)} d \eta\right.} \\
& =e^{-i b \cdot \xi}(2 \pi)^{-n / 2} \int_{\mathbb{R}^{n}} e^{-i b \cdot \eta} \hat{f}(\eta) \overline{\hat{\varphi}\left(\left(A_{\xi}^{-1}\right)^{t}(\eta-\xi)\right)} d \eta \\
& =e^{-i b \cdot \xi}\left(\mathcal{F}^{-1} f_{\xi, A_{\xi}}(b),\right.
\end{aligned}
$$

as required.

We can now give a continuous inversion formula for the $n$-dimensional Stockwell transform. 
Theorem 2.3. Let $A_{\xi}$ be a matrix such that

(i) $A_{\xi} \in \mathrm{GL}(n, \mathbb{R})$ for every $\xi \in \mathbb{R}^{n} \backslash\{0\}$,

(ii) if $\eta=\left(A_{\xi}^{-1}\right)^{t} \zeta$ for some $\zeta \in \mathbb{R}^{n}$, then there exist positive functions $f_{1}, f_{2}: \mathbb{R}^{n} \rightarrow \mathbb{R}$ for which

$$
\left|\operatorname{det}\left(\frac{\partial \eta}{\partial \xi}\right)\right|=\frac{f_{1}(\eta)}{f_{2}(\xi)}, \quad \xi, \eta \in \mathbb{R}^{n}
$$

where $\frac{\partial \eta}{\partial \xi}$ is the Jacobian matrix of $\eta$ with respect to $\xi$,

(iii) there exists a vector $v \in \mathbb{R}^{n}$ for which

$$
\left(A_{\xi}^{-1}\right)^{t} \xi=v
$$

Let $\varphi \in L^{1}\left(\mathbb{R}^{n}\right) \cap L^{2}\left(\mathbb{R}^{n}\right)$ be such that

$$
c_{\varphi}=\int_{\mathbb{R}^{n}}|\widehat{\varphi}(\eta-v)|^{2} \frac{d \eta}{f_{1}(\eta)}<\infty .
$$

Let $S_{\varphi}$ be the associated Stockwell transform, i.e.,

$$
\left(S_{\varphi} f\right)(b, \xi)=(2 \pi)^{-n / 2}\left|\operatorname{det} A_{\xi}\right| \int_{\mathbb{R}^{n}} e^{-i x \cdot \xi} f(x) \overline{\varphi\left(A_{\xi}(x-b)\right)} d x, \quad b \in \mathbb{R}^{n}, \xi \in \mathbb{R}^{n} \backslash\{0\} .
$$

Then for every $f$ and $g$ in $L^{2}\left(\mathbb{R}^{n}\right)$, we have

$$
\int_{\mathbb{R}^{n}} \int_{\mathbb{R}^{n}}\left(S_{\varphi} f\right)(b, \xi) \overline{\left(S_{\varphi} g\right)(b, \xi)} d b \frac{d \xi}{f_{2}(\xi)}=c_{\varphi}(f, g)_{L^{2}\left(\mathbb{R}^{n}\right)} .
$$

To give a proof of Theorem 2.3, we need the following lemma.

Lemma 2.4. Let $A_{\xi}$ be a matrix satisfying (i), (ii) and (iii) of Theorem 2.3. Then, for all $\varphi \in L^{2}\left(\mathbb{R}^{n}\right)$,

$$
\int_{\mathbb{R}^{n}}\left|\widehat{\varphi}\left(\left(A_{\xi}^{-1}\right)^{t}(\zeta-\xi)\right)\right|^{2} \frac{d \xi}{f_{2}(\xi)}=\int_{\mathbb{R}^{n}}|\widehat{\varphi}(\eta-v)|^{2} \frac{d \eta}{f_{1}(\eta)} .
$$

Proof. Let $\eta=\left(A_{\xi}^{-1}\right)^{t} \zeta$. Then by (ii), there exist positive functions $f_{1}, f_{2}: \mathbb{R}^{n} \rightarrow \mathbb{R}$ such that for all $\xi$ and $\eta$ in $\mathbb{R}^{n}$,

$$
\left|\operatorname{det}\left(\frac{\partial \eta}{\partial \xi}\right)\right|=\frac{f_{1}(\eta)}{f_{2}(\xi)} .
$$

So,

$$
d \eta=\left|\operatorname{det}\left(\frac{\partial \eta}{\partial \xi}\right)\right| d \xi=\frac{f_{1}(\eta)}{f_{2}(\xi)} d \xi
$$

Using (iii),

$$
\begin{aligned}
\int_{\mathbb{R}^{n}}\left|\widehat{\varphi}\left(\left(A_{\xi}^{-1}\right)^{t}(\zeta-\xi)\right)\right|^{2} \frac{d \xi}{f_{2}(\xi)} & =\int_{\mathbb{R}^{n}} \mid \widehat{\varphi}\left(\left.\left(A_{\xi}^{-1}\right)^{t}(\zeta-v)\right|^{2} \frac{d \xi}{f_{2}(\xi)}\right. \\
& =\int_{\mathbb{R}^{n}}|\widehat{\varphi}(\eta-v)|^{2} \frac{d \eta}{f_{1}(\eta)}
\end{aligned}
$$


Proof of Theorem 2.3. By Proposition 2.2, Lemma 2.4 and Plancherel's formula, we have

$$
\begin{aligned}
& \int_{\mathbb{R}^{n}} \int_{\mathbb{R}^{n}}\left(S_{\varphi} f\right)(b, \xi) \overline{\left(S_{\varphi} g\right)(b, \xi)} d b \frac{d \xi}{f_{2}(\xi)} \\
= & \int_{\mathbb{R}^{n}} \int_{\mathbb{R}^{n}} \hat{f}(\zeta) \overline{\hat{g}(\zeta)}\left|\hat{\varphi}\left(\left(A_{\xi}^{-1}\right)^{t}(\zeta-\xi)\right)\right|^{2} d \zeta \frac{d \xi}{f_{2}(\xi)} \\
= & \int_{\mathbb{R}^{n}} \hat{f}(\zeta) \overline{\hat{g}(\zeta)}\left(\int_{\mathbb{R}^{n}}\left|\hat{\varphi}\left(\left(A_{\xi}^{-1}\right)^{t}(\zeta-\xi)\right)\right|^{2} \frac{d \xi}{f_{2}(\xi)}\right) d \zeta \\
= & \int_{\mathbb{R}^{n}} \hat{f}(\zeta) \overline{\hat{g}(\zeta)}\left(\int_{\mathbb{R}^{n}}|\hat{\varphi}(\eta-v)|^{2} \frac{d \eta}{f_{1}(\eta)}\right) d \zeta \\
= & c_{\varphi} \int_{\mathbb{R}^{n}} \hat{f}(\zeta) \overline{\hat{g}(\zeta)} d \zeta \\
= & c_{\varphi}(f, g)_{L^{2}\left(\mathbb{R}^{n}\right)} .
\end{aligned}
$$

\section{Multi-Dimensional Gabor Transforms}

Let $A$ be an invertible $n \times n$ matrix with real entries, and let $\varphi$ be a function in $L^{1}\left(\mathbb{R}^{n}\right) \cap L^{2}\left(\mathbb{R}^{n}\right)$. Then the simplest $n$-dimensional Stockwell transform $S_{\varphi} f$ of a signal $f$ in $L^{2}\left(\mathbb{R}^{n}\right)$ is given by

$$
\left(S_{\varphi} f\right)(b, \xi)=(2 \pi)^{-n / 2}|\operatorname{det} A| \int_{\mathbb{R}^{n}} e^{-i x \cdot \xi} f(x) \overline{\varphi(A(x-b))} d x
$$

for all $b, \xi$ in $\mathbb{R}^{n}$.

It is easy to see that the preceding $n$-dimensional Stockwell transform in which the matrix $A$ is independent of $\xi$ in $\mathbb{R}^{n}$ can be looked at as the $n$-dimensional Gabor transform.

The following result is the analog of Theorem 1.1 for $n$-dimensional Gabor transforms.

Theorem 3.1. Let $\varphi$ be a function in $L^{1}\left(\mathbb{R}^{n}\right) \cap L^{2}\left(\mathbb{R}^{n}\right)$ such that

$$
\|\varphi\|_{2}=1 \text {. }
$$

Then for all $f$ and $g$ in $L^{2}\left(\mathbb{R}^{n}\right)$,

$$
(f, g)_{L^{2}\left(\mathbb{R}^{n}\right)}=\int_{\mathbb{R}^{n}} \int_{\mathbb{R}^{n}}\left(S_{\varphi} f\right)(b, \xi) \overline{\left(S_{\varphi} g\right)(b, \xi)} d b \frac{d \xi}{|\operatorname{det} A|} .
$$

Proof. By Proposition 2.2 and Plancherel's formula, we have

$$
\begin{aligned}
& \int_{\mathbb{R}^{n}} \int_{\mathbb{R}^{n}}\left(S_{\varphi} f\right)(b, \xi) \overline{\left(S_{\varphi} g\right)(b, \xi)} \frac{d b d \xi}{|\operatorname{det} A|} \\
= & \int_{\mathbb{R}^{n}} \int_{\mathbb{R}^{n}} \hat{f}(\eta) \overline{\hat{g}(\eta)}\left|\hat{\varphi}\left(\left(A^{-1}\right)^{t}(\eta-\xi)\right)\right|^{2} \frac{d \eta d \xi}{|\operatorname{det} A|} \\
= & \int_{\mathbb{R}^{n}} \hat{f}(\eta) \overline{\hat{g}(\eta)}\left[\int_{\mathbb{R}^{n}} \mid \hat{\varphi}\left(\left.\left(A^{-1}\right)^{t}(\eta-\xi)\right|^{2} \frac{d \xi}{|\operatorname{det} A|}\right] d \eta\right. \\
= & \int_{\mathbb{R}^{n}} \hat{f}(\eta) \overline{\hat{g}(\eta)}\left[\int_{\mathbb{R}^{n}}|\hat{\varphi}(\zeta)|^{2} d \zeta\right] d \eta \\
= & \int_{\mathbb{R}^{n}} \hat{f}(\eta) \overline{\hat{g}(\eta)} d \eta \\
= & (f, g)_{L^{2}\left(\mathbb{R}^{n}\right) .}
\end{aligned}
$$




\section{Multi-Dimensional Non-Isotropic Stockwell Transforms}

For all $\xi=\left(\xi_{1}, \xi_{2}, \ldots, \xi_{n}\right)$ in $\mathbb{R}^{n}$ with

$$
\xi_{j} \neq 0
$$

for $j=1,2, \ldots, n$, we consider the diagonal matrix $I_{\xi}$ given by

$$
I_{\xi}=\left(\begin{array}{cccccc}
\xi_{1} & 0 & 0 & \cdots & 0 & 0 \\
0 & \xi_{2} & 0 & \cdots & 0 & 0 \\
\vdots & \vdots & \vdots & \vdots & \vdots & \vdots \\
0 & 0 & 0 & \cdots & 0 & \xi_{n}
\end{array}\right)
$$

Let $\varphi \in L^{1}\left(\mathbb{R}^{n}\right) \cap L^{2}\left(\mathbb{R}^{n}\right)$ be such that

$$
\int_{\mathbb{R}^{n}} \varphi(x) d x=1 .
$$

Then the non-isotropic $n$-dimensional Stockwell transform $S_{\varphi} f$ of a signal $f$ in $L^{2}\left(\mathbb{R}^{n}\right)$ is given by

$$
\begin{aligned}
\left(S_{\varphi} f\right)(b, \xi) & =(2 \pi)^{-n / 2}\left|\operatorname{det} I_{\xi}\right| \int_{\mathbb{R}^{n}} e^{-i x \cdot \xi} f(x) \overline{\varphi\left(I_{\xi}(x-b)\right)} d x \\
& =(2 \pi)^{-n / 2} \prod_{j=1}^{n}\left|\xi_{j}\right| \int_{\mathbb{R}^{n}} e^{-i x \cdot \xi} f(x) \overline{\varphi\left(I_{\xi}(x-b)\right)} d x
\end{aligned}
$$

for all $b$ in $\mathbb{R}^{n}$ and all $\xi$ in $\mathbb{R}^{n} \backslash\{0\}$.

Corollary 4.1. Let $\varphi \in L^{1}\left(\mathbb{R}^{n}\right) \cap L^{2}\left(\mathbb{R}^{n}\right)$ be such that

$$
\int_{\mathbb{R}^{n}}|\hat{\varphi}(\xi-\mathbf{1})|^{2} \frac{d \xi}{\prod_{j=1}^{n}\left|\xi_{j}\right|}<\infty
$$

where $\mathbf{1}=(1,1, \ldots, 1) \in \mathbb{R}^{n}$. Then for all $f$ and $g$ in $L^{2}\left(\mathbb{R}^{n}\right)$,

$$
(f, g)_{L^{2}\left(\mathbb{R}^{n}\right)}=\frac{1}{c_{\varphi}} \int_{\mathbb{R}^{n}} \int_{\mathbb{R}^{n}}\left(S_{\varphi} f\right)(b, \xi) \overline{\left(S_{\varphi} g\right)(b, \xi)} \frac{d b d \xi}{\prod_{j=1}^{n}\left|\xi_{j}\right|},
$$

where

$$
c_{\varphi}=\int_{\mathbb{R}^{n}}|\hat{\varphi}(\xi-\mathbf{1})|^{2} \frac{d \xi}{\prod_{j=1}^{n}\left|\xi_{j}\right|} .
$$

Proof. It is not hard to check that $I_{\xi}$ satisfies (i) and (iii) of Theorem 2.3, with

$$
v=\mathbf{1}=(1,1, \ldots, 1) \in \mathbb{R}^{n} .
$$

Furthermore, choosing $\eta=\left(I_{\xi}^{-1}\right)^{t} \zeta$, then for all $\eta$ in $\mathbb{R}^{n}$ and all $\xi$ in $\mathbb{R}^{n}$ with

$$
\xi_{j} \neq 0, \quad j=1,2, \ldots, n,
$$

we get

$$
\left|\operatorname{det}\left(\frac{\partial \eta}{\partial \xi}\right)\right|=\prod_{j=1}^{n}\left|\eta_{j}\right| /\left|\xi_{j}\right|,
$$

so $I_{\xi}$ satisfies (ii) of Theorem 2.3 with

$$
f_{1}(\eta)=\prod_{j=1}^{n}\left|\eta_{j}\right|
$$


and

$$
f_{2}(\xi)=\prod_{j=1}^{n}\left|\xi_{j}\right| .
$$

It should be noted that Theorem 3.1 and hence Corollary 4.1 are simple consequences of the unitarity of the transform $|\operatorname{det} A|^{1 / 2} S_{\varphi}$.

\section{Special Matrices Related to the Stockwell Transforms}

In this section we introduce a special class of matrices satisfying the hypotheses of Theorem 2.3.

Theorem 5.1. Let $A_{\xi}$ be a matrix such that

(a) $\frac{1}{|\xi|} A_{\xi} \in \mathrm{O}(n, \mathbb{R})$ for every $\xi \in \mathbb{R}^{n} \backslash\{0\}$,

(b) there exists a matrix $P \in \mathrm{O}(n, \mathbb{R})$ for which $A_{\xi} \zeta=P A_{\zeta} \xi$,

(c) there exists a vector $v \in \mathbb{R}^{n}$ for which

$$
\left(A_{\xi}^{-1}\right)^{t} \xi=v .
$$

Let $\varphi \in L^{1}\left(\mathbb{R}^{n}\right) \cap L^{2}\left(\mathbb{R}^{n}\right)$ be such that

$$
c_{\varphi}=\int_{\mathbb{R}^{n}}|\widehat{\varphi}(\eta-v)|^{2} \frac{d \eta}{f_{1}(\eta)}<\infty .
$$

Let $S_{\varphi}$ the associated Stockwell transform, i.e.,

$$
\left(S_{\varphi} f\right)(b, \xi)=(2 \pi)^{-n / 2}\left|\operatorname{det} A_{\xi}\right| \int_{\mathbb{R}^{n}} e^{-i x \cdot \xi} f(x) \overline{\varphi\left(A_{\xi}(x-b)\right)} d x, \quad b \in \mathbb{R}^{n}, \xi \in \mathbb{R}^{n} \backslash\{0\} .
$$

Then for every $f$ and $g$ in $L^{2}\left(\mathbb{R}^{n}\right)$, we have

$$
\int_{\mathbb{R}^{n}} \int_{\mathbb{R}^{n}}\left(S_{\varphi} f\right)(b, \xi) \overline{\left(S_{\varphi} g\right)(b, \xi)} d b \frac{d \xi}{f_{2}(\xi)}=c_{\varphi}(f, g)_{L^{2}\left(\mathbb{R}^{n}\right)} .
$$

The proof is a direct consequence of the following lemma.

Lemma 5.2. Let $A_{\xi}$ be a matrix such that

(a) $\frac{1}{|\xi|} A_{\xi} \in \mathrm{O}(n, \mathbb{R})$ for every $\xi \in \mathbb{R}^{n} \backslash\{0\}$,

(b) there exists a matrix $P \in \mathrm{O}(n, \mathbb{R})$ for which $A_{\xi} \zeta=P A_{\zeta} \xi$.

Then $A_{\xi}$ satisfies (i) and (ii) of Theorem 2.3.

Proof. Clearly (a) implies (i), so it is sufficient to prove that (b) implies (ii). To do this, we want to show that given $\eta=\left(A_{\xi}^{-1}\right)^{t} \zeta$, then

$$
\left|\operatorname{det}\left(\frac{\partial \eta}{\partial \xi}\right)\right|=\frac{|\eta|^{n}}{|\xi|^{n}}
$$

It is easy to show that (a) implies that

(1) $\left(A_{\xi}^{-1}\right)^{t}=\frac{1}{|\xi|^{2}} A_{\xi}$ for every $\xi \in \mathbb{R}^{n} \backslash\{0\}$,

(2) $\left|\operatorname{det} A_{\xi}\right|=|\xi|^{n}$ for every $\xi \in \mathbb{R}^{n} \backslash\{0\}$. 
In fact, using (a), we have

$$
\left(A_{\xi}^{-1}\right)^{t}=\left(\left(|\xi| \frac{1}{|\xi|} A_{\xi}\right)^{-1}\right)^{t}=\frac{1}{|\xi|}\left(\left(\frac{1}{|\xi|} A_{\xi}\right)^{-1}\right)^{t}=\frac{1}{|\xi|^{2}} A_{\xi}
$$

and

$$
\left|\operatorname{det} A_{\xi}\right|=\left|\operatorname{det}\left(|\xi| \frac{1}{|\xi|} A_{\xi}\right)\right|=|\xi|^{n}\left|\operatorname{det}\left(\frac{1}{|\xi|} A_{\xi}\right)\right|=|\xi|^{n} .
$$

Let us set $\eta=\frac{1}{|\xi|^{2}} A_{\xi} \zeta$ and notice that

$$
|\eta|=\left|\frac{1}{|\xi|}\left(\frac{1}{|\xi|} A_{\xi} \zeta\right)\right|=\frac{|\zeta|}{|\xi|}
$$

Then

$$
|\zeta|=|\eta||\xi|
$$

Furthermore, by (b)

$$
\eta=\frac{1}{|\xi|^{2}} A_{\xi} \zeta=\frac{1}{|\xi|^{2}} P A_{\zeta} \xi=P A_{\zeta} \frac{\xi}{|\xi|^{2}},
$$

so, for all $\eta$ in $\mathbb{R}^{n}$ and $\xi \in \mathbb{R}^{n} \backslash\{0\}$,

$$
\begin{aligned}
J_{\eta} & =P A_{\zeta}\left(\begin{array}{cccc}
\frac{|\xi|^{2}-2 \xi_{1}^{2}}{|\xi|^{4}} & \frac{-2 \xi_{1} \xi_{2}}{|\xi|^{4}} & \ldots & \frac{-2 \xi_{1} \xi_{n}}{|\xi|^{4}} \\
\frac{-2 \xi_{2} \xi_{1}}{|\xi|^{4}} & \frac{|\xi|^{2}-2 \xi_{2}^{2}}{|\xi|^{4}} & \cdots & \frac{-2 \xi_{2} \xi_{n}}{|\xi|^{4}} \\
\vdots & \vdots & \vdots & \vdots \\
\frac{-2 \xi_{n} \xi_{1}}{|\xi|^{4}} & \frac{-2 \xi_{n} \xi_{2}}{|\xi|^{4}} & \cdots & \frac{|\xi|^{2}-2 \xi_{n}^{2}}{|\xi|^{4}}
\end{array}\right) \\
& =\frac{1}{|\xi|^{4}} P A_{\zeta}\left(|\xi|^{2} I-2 C_{\xi}\right) \\
& =\frac{1}{|\xi|^{2}} P A_{\zeta}\left(I-\frac{2}{|\xi|^{2}} C_{\xi}\right),
\end{aligned}
$$

where

$$
C_{\xi}=\left(\begin{array}{cccc}
\xi_{1}^{2} & \xi_{1} \xi_{2} & \cdots & \xi_{1} \xi_{n} \\
\xi_{2} \xi_{1} & \xi_{2}^{2} & \cdots & \xi_{2} \xi_{n} \\
\vdots & \vdots & \vdots & \vdots \\
\xi_{n} \xi_{1} & \xi_{n} \xi_{2} & \cdots & \xi_{n}^{2}
\end{array}\right)
$$

and $I$ is the $n$-dimensional identity matrix. At this point it is useful to prove that

$$
\operatorname{det}\left(I-\frac{2}{|\xi|^{2}} C_{\xi}\right)=-1
$$

To do this, first observe that $\operatorname{rank} C_{\xi}=1, C_{\xi}$ is a symmetric matrix, so there exists only one nonzero eigenvalue. We can check that $|\xi|^{2}$ is the only nonzero eigenvalue associated to the eigenvector $\left(\xi_{1}, \xi_{2}, \ldots, \xi_{n}\right)$. 
In fact

$$
\begin{aligned}
C_{\xi}\left(\begin{array}{c}
\xi_{1} \\
\xi_{2} \\
\vdots \\
\xi_{n}
\end{array}\right) & =\left(\begin{array}{cccc}
\xi_{1}^{2} & \xi_{1} \xi_{2} & \ldots & \xi_{1} \xi_{n} \\
\xi_{2} \xi_{1} & \xi_{2}^{2} & \cdots & \xi_{2} \xi_{n} \\
\vdots & \vdots & \vdots & \vdots \\
\xi_{n} \xi_{1} & \xi_{n} \xi_{2} & \vdots & \xi_{n}^{2}
\end{array}\right)\left(\begin{array}{c}
\xi_{1} \\
\xi_{2} \\
\vdots \\
\xi_{n}
\end{array}\right) \\
& =\left(\begin{array}{ccccc}
\xi_{1} & 0 & \cdots & 0 \\
0 & \xi_{2} & \cdots & 0 \\
\vdots & \vdots & \vdots & \vdots \\
0 & 0 & \cdots & \xi_{n}
\end{array}\right)\left(\begin{array}{cccc}
\xi_{1} & \xi_{2} & \ldots & \xi_{n} \\
\xi_{1} & \xi_{2} & \ldots & \xi_{n} \\
\vdots & \vdots & \vdots & \vdots \\
\xi_{1} & \xi_{2} & \cdots & \xi_{n}
\end{array}\right)\left(\begin{array}{c}
\xi_{1} \\
\xi_{2} \\
\vdots \\
\xi_{n}
\end{array}\right) \\
& =|\xi|^{2}\left(\begin{array}{cccc}
\xi_{1} & 0 & \ldots & 0 \\
0 & \xi_{2} & \cdots & 0 \\
\vdots & \vdots & \vdots & \vdots \\
0 & 0 & \cdots & \xi_{n}
\end{array}\right)\left(\begin{array}{c}
1 \\
1 \\
\xi_{1} \\
\xi_{2} \\
\vdots \\
1
\end{array}\right)=|\xi|^{2}\left(\begin{array}{c}
\xi_{n} \\
\xi_{n}
\end{array}\right)
\end{aligned}
$$

So, the eigenvalues $\lambda_{1}^{C}, \ldots, \lambda_{n}^{C}$ of $-\frac{2}{|\xi|^{2}} C_{\xi}$ are $\lambda_{1}^{C}=\cdots=\lambda_{n-1}^{C}=0$ and $\lambda_{n}^{C}=-2$. We introduce

$$
B_{\xi}=I-\frac{2}{|\xi|^{2}} C_{\xi},
$$

and we compute the eigenvalues $\lambda^{B}$ of $B_{\xi}$ by studying its characteristic equation, i.e.,

$$
0=\operatorname{det}\left(\lambda^{B} I-B_{\xi}\right)=\operatorname{det}\left(\lambda^{B} I-I+\frac{2}{|\xi|^{2}} C_{\xi}\right)=\operatorname{det}\left(\left(\lambda^{B}-1\right) I-\left(-\frac{2}{|\xi|^{2}} C_{\xi}\right)\right) .
$$

It follows that the eigenvalues of $B_{\xi}$ are given by

$$
\lambda_{j}^{B}=\lambda_{j}^{C}+1, \quad j=1, \ldots, n .
$$

So, $\lambda_{1}^{B}=\cdots=\lambda_{n-1}^{B}=1$ and $\lambda_{n}^{B}=-1$ and it is sufficient to observe that

$$
\operatorname{det}\left(I-\frac{2}{|\xi|^{2}} C_{\xi}\right)=\operatorname{det} B_{\xi}=\prod_{j=1}^{n} \lambda_{j}^{B}=-1 .
$$

Now, by the preceding observations,

$$
\begin{aligned}
\left|\operatorname{det}\left(\frac{\partial \eta}{\partial \xi}\right)\right| & =\operatorname{det}\left(\frac{1}{|\xi|^{2}} P A_{\zeta}\left(I-\frac{2}{|\xi|^{2}} C_{\xi}\right)\right) \\
& =\operatorname{det}\left(\frac{1}{|\xi|^{2}} P A_{\zeta}\right) \operatorname{det}\left(I-\frac{2}{|\xi|^{2}} C_{\xi}\right) \\
& =-\frac{1}{|\xi|^{2 n}} \operatorname{det}\left(P A_{\zeta}\right) \\
& =-\frac{1}{|\xi|^{2 n}}(\operatorname{det} P)\left(\operatorname{det} A_{\zeta}\right) .
\end{aligned}
$$

Finally, by (b) and (5.1),

$$
\left|\operatorname{det}\left(\frac{\partial \eta}{\partial \xi}\right)\right|=\left|-\frac{1}{|\xi|^{2 n}}(\operatorname{det} P)\left(\operatorname{det} A_{\zeta}\right)\right|=\frac{1}{|\xi|^{2 n}}|\zeta|^{n}=\frac{|\eta|^{n}}{|\xi|^{n}} .
$$


Example 5.3. The following matrices are examples of $2 \times 2,4 \times 4$ and $8 \times 8$ matrices satisfying the hypotheses of Theorem 5.1.

$$
\begin{aligned}
A_{\xi}^{2 \times 2}= & \left(\begin{array}{cc}
\xi_{1} & \xi_{2} \\
-\xi_{2} & \xi_{1}
\end{array}\right), \\
A_{\xi}^{4 \times 4}= & \left(\begin{array}{ccccccc}
\xi_{1} & \xi_{2} & \xi_{3} & \xi_{4} \\
-\xi_{2} & \xi_{1} & \xi_{4} & -\xi_{3} \\
-\xi_{3} & -\xi_{4} & \xi_{1} & \xi_{2} \\
-\xi_{4} & \xi_{3} & -\xi_{2} & \xi_{1}
\end{array}\right), \\
A_{\xi}^{8 \times 8}= & \left(\begin{array}{cccccccc}
\xi_{1} & \xi_{2} & \xi_{3} & \xi_{4} & \xi_{5} & \xi_{6} & \xi_{7} & \xi_{8} \\
-\xi_{2} & \xi_{1} & \xi_{4} & -\xi_{3} & \xi_{6} & -\xi_{5} & -\xi_{8} & \xi_{7} \\
-\xi_{3} & -\xi_{4} & \xi_{1} & \xi_{2} & \xi_{7} & \xi_{8} & -\xi_{5} & -\xi_{6} \\
-\xi_{4} & \xi_{3} & -\xi_{2} & \xi_{1} & \xi_{8} & -\xi_{7} & \xi_{6} & -\xi_{5} \\
-\xi_{5} & -\xi_{6} & -\xi_{7} & -\xi_{8} & \xi_{1} & \xi_{2} & \xi_{3} & \xi_{4} \\
-\xi_{6} & \xi_{5} & -\xi_{8} & \xi_{7} & -\xi_{2} & \xi_{1} & -\xi_{4} & \xi_{3} \\
-\xi_{7} & \xi_{8} & \xi_{5} & -\xi_{6} & -\xi_{3} & \xi_{4} & \xi_{1} & -\xi_{2} \\
-\xi_{8} & -\xi_{7} & \xi_{6} & \xi_{5} & -\xi_{4} & -\xi_{3} & \xi_{2} & \xi_{1}
\end{array}\right) .
\end{aligned}
$$

\section{Conclusions}

We have given in this paper continuous inversion formulas for $n$-dimensional Stockwell transforms that include the $n$-dimensional Gabor transforms as special cases. The impetus for introducing these $n$ dimensional analogs comes from the needs to deal with data that depend on many parameters and the future possibility to process images in modern physics in which dimensions more than four are being envisaged. The techniques in this paper should be studied and used with other techniques in, e.g., $[2,5,15]$. Furthermore, the Stockwell transforms, like the Gabor transforms, are the backbones of filters, also known as localization operators in [16], which are of great interests in the analysis of signals and images.

Acknowledgements. The authors are grateful to the referee for the very useful comments, which have been incorporated into the paper.

\section{References}

[1] J.-P. Antoine, R. Murenzi, P. Vandergheynst, S. T. Ali. Two-Dimensional Wavelets and their Relatives. Cambridge University Press, 2004.

[2] L. Cohen. Time-Frequency Analysis. Prentice Hall, 1995.

[3] E. Cordero, F. De Mari, K. Nowak, A. Tabacco. Analytic features of reproducing groups for the metaplectic representation. J. Fourier Anal. Appl. 12 (2006), 157-180.

[4] A. Cordoba, C. Fefferman. Wave packets and Fourier integral operators. Comm. Partial Differential Equations 3 (1978), 979-1005.

[5] I. Daubechies. Ten Lectures on Wavelets. SIAM, 1992.

[6] M. G. Eramian, R. A. Schincariol, L. Mansinha, R. G. Stockwell. Generation of aquifer heterogeneity maps using two dimensional spectral texture segmentation techniques. Math. Geology 31 (1999), 327-348.

[7] G. B. Folland. Harmonic Analysis in Phase Space. Princeton University Press, 1989.

[8] B. G. Goodyear, H. Zhu, R. A. Brown, J. R. Mitchell. Removal of phase artifacts from fMRI data using a Stockwell transform filter improves brain activity detection. Magn. Reson. Med. 51 (2004), 16-21.

[9] A. Grossmann, J. Morlet. Decomposition of Hardy functions into square integrable wavelets of constant shape SIAM J. Math. Anal. 15 (1984), 723-736.

[10] Q. Guo, S. Molahajloo, M. W. Wong. Modified Stockwell transforms and time-frequency analysis in New Developments in Pseudo-Differential Operators. Operator Theory: Advances and Applications 189, Birkhäuser, 2009, $275-285$.

[11] Q. Guo, M. W. Wong. Modified Stockwell transforms, Memorie della Accademia delle Scienze di Torino, Classe di Scienze, Fische. Matematiche e Naturali, Serie V, Vol. 32 (2008), 3-20.

[12] Y. Liu and M. W. Wong. Inversion formulas for two-dimensional Stockwell transforms, in Pseudo-Differential Operators: Partial Differential Equations and Time-Frequency Analysis. Fields Institute Communications 52, American Mathematical Society, 2007, 323-330. 
[13] R. G. Stockwell, L. Mansinha, R. P. Lowe. Localization of the complex spectrum: the S transform. IEEE Trans. Signal Processing 44 (1996), 998-1001.

[14] D. Bernier, K. F. Taylor. Wavelets from square-integrable representations. SIAM J. Math. Anal. 27 (1996), $594-608$.

[15] M. W. Wong. Weyl Transforms. Springer, 1998.

[16] M. W. Wong. Wavelet Transforms and Localization Operators. Birkhäuser, 2002.

[17] M. W. Wong, H. Zhu. A characterization of the Stockwell spectrum, in Modern Trends in Pseudo-Differential Operators. Birkhäuser, 2007, 251-257.

[18] H. Zhu, B. G. Goodyear, M. L. Lauzon, R. A. Brown, G. S. Mayer, L. Mansinha, A. G. Law, J. R. Mitchell. A new multiscale Fourier analysis for MRI. Med. Phys. 30 (2003), 1134-1141. 